\title{
Similar eficacia de la monoterapia con bupropion, sertralina y venlafaxina en pacientes con depresión que no habian respondido a citalopram
}

Similar eficacy of bupropion, sertraline and venlafaxine monotherapy in in depressed patients with no response to citalopram as first line treatment

STAR*D Study Team. N Engl J Med. 2006;354(12):1231-42

\section{Objetivo}

Evaluar efectividad del cambio a bupropion, sertralina o venlafaxina como segunda línea en el tratamiento de la depresión.

\section{Diseño}

Ensayo clínico, aleatorizado, controlado.

Lugar

28 centros primarios y 23 psiquiátricos. Universidad de Texas. EE.UU.

\section{Participantes}

727 adultos ambulatorios con trastorno depresivo mayor no psicótico que no tuvieron remisión o no toleraron el citalopram como primera opción de tratamiento luego de 12 semanas. Fueron aleatorizados a cambiar de antidepresivo el cual recibieron durante 14 semanas (239 bupropion, 238 sertralina, 250 venlafaxina).

\section{Medición de resultados principales}

El objetivo del tratamiento fue la remisión, definida como una puntuación de cinco o menos de los 16 ítems del Inventario Rápido Autoevaluado de Sintomatología Depresiva (QIDS-C-16) o una puntuación de siete o menos en la Escala de Hamilton para la Depresión (HRSD-17). El citalopram fue discontinuado sin período de disminución. Las dosis iniciales y subsecuentes pueden verse en la tabla 1.

Tabla 1: esquema de dosificación de cada droga en mg/día.

\begin{tabular}{l|c|c|c|c|c|c}
\hline & $\begin{array}{c}\text { Dosis } \\
\text { inicial }\end{array}$ & $\begin{array}{c}\text { Día } \\
\mathbf{8} \mathbf{1 4}\end{array}$ & $\begin{array}{c}\text { Día } \\
\mathbf{1 5} \mathbf{a} \mathbf{2 7}\end{array}$ & $\begin{array}{c}\mathbf{D} \text { D́a } \\
\mathbf{2 8} \text { a } \mathbf{4 1}\end{array}$ & $\begin{array}{c}\text { Dáa } \\
\mathbf{4 2} \text { a } \mathbf{6 3}\end{array}$ & $\begin{array}{c}\mathbf{6 3} \text { en } \\
\text { adelante }\end{array}$ \\
\hline Bupropion & 150 & 200 & 200 & 300 & 400 & 400 \\
\hline Sertralina & 50 & 50 & 100 & 150 & 150 & 200 \\
\hline Venlafaxina & 37,5 & 75 & 150 & 225 & 300 & 375 \\
\hline
\end{tabular}

Las drogas usadas como segunda línea son diferentes entre sí. El bupropion bloquea la recaptación de dopamina y norepinefrina; la sertralina es un inhibidor selectivo de la recaptacion de serotonina (IRSS) y la venlafaxina es un agente dual, que bloquea la recaptación de serotonina y de noradrenalina.

\section{Resultados principales}

Tanto las tasas de remisión y de respuesta, como el tiempo a las mismas no difirieron significativamente entre las distintas ramas. Ver tabla 2. La tolerabilidad y los efectos adversos fueron similares en los tres grupos.

Tabla 2: tasas de remisión y respuesta y tiempo a las mismas de acuerdo al tratamiento instituido en reemplazo de citalopram en pacientes con depresión que no habían respondido a esta droga.

\begin{tabular}{|c|c|c|c|c|}
\hline & \multicolumn{2}{|c|}{ Tasa de remisión } & \multirow{2}{*}{$\begin{array}{l}\text { Tasa de } \\
\text { respuesta }\end{array}$} & \multirow{2}{*}{$\begin{array}{l}\text { Tiempo a la remisión } \\
\text { en semanas }\end{array}$} \\
\hline & Hamilton & QIDS-C-16 & & \\
\hline Bupropion & $21,3 \%$ & $25,5 \%$ & $26,1 \%$ & $5,1-4,5$ \\
\hline Sertralina & $17,6 \%$ & $26 \%$ & $26.7 \%$ & $6,2-5$ \\
\hline Venlafaxina & $24,8 \%$ & $25 \%$ & $28,2 \%$ & $5,5-4,7$ \\
\hline
\end{tabular}

\section{Conclusiones}

En pacientes sin respuesta previa a citalopram, las tasas de remisión son de aproximadamente $25 \%$, independientemente de la droga elegida en su reemplazo. La intolerancia o falta de respuesta a un IRRS no predice la ineficacia o intolerancia a otra droga de la misma familia

Palabras claves: Depresión, fracaso tratamiento, antidepresivos. Key words: depression, treatment failure, antidepresives.

Fuente de financiamiento: Instituto de salud mental del Instituto de Salud de Estados Unidos.

\section{Conclusiones del comentador}

EISTAR*D es un estudio valioso para la práctica clínica, por la cantidad de pacientes involucrados y el tiempo total de seguimiento. Ayuda a desmitificar ciertos preceptos arraigados en la práctica y provee una conclusión desalentadora: sólo $30 \%$ de los pacientes tuvieron remisión al cabo del de la primera línea de tratamiento. Si tenemos en cuenta que el tiempo de retraso antes de recibir el primer tratamiento de depresión es en promedio seis a ocho años, las posibilidades actuales de obtener rápida remisión sintomática son aún más sombrías.

Gabriela La Monica. [ Especialista en Psiquiatría y Psicología médica. La Plata. Buenos Aires. Argentina. ]

La Monica G. Tratamiento de la Depresión: El agregado de bupropion tiene la misma eficacia que el de buspirona en pacientes deprimidos que no habían respondido a citalopram. Similar eficacia de la monoterapia con bupropion, sertralina y venlafaxina en pacientes con depresión que no habían respondido a cital anbul; 10(1):10-11, ene-feb.2007. Comentados de: Trivedi MH, Fava M, Wisniewski SR et al; STAR*D Study Team. citalopram. Evid. actual. práct. ambul; 10(1):10-11, ene-feb.2007. Comentados de: Trivedi MH, Fava M, Wisniewsion Wisniewski SR, et al; STAR*D Study Team. Bupropion-SR, sertraline, or venlafaxine-XR after failure of SSRIs for depression. N Engl J Med. 2006;354(12):123142. PMID: 16554525

\section{Referencias}

1. Murray CJL, Lopez AD: Global Health Statistics: a compendium of incidence, prevalence and mortality estimates for over 200 conditions. Cambridge MA Harvard University Press on behalf of the World Health Organization and the World Bank; 1996.

2. Rojtenberg S. (2001). Depresiones y antidepresivos. Ed Medica Panamericana.

3. Simon GE; VonKorff M. Recognition, management, and outcomes of depression in primary care. Arch Fam Med 1995 Feb;4(2):99-105.

4. Depression Guideline Panel. Depression in Primary Care: Volume 1. Detection and Diagnosis. Clinical Practice Guideline, Number 5. Rockville, MD: U.S Department of Health and Human Services; 1993. AHCPR Publication No. 93-0550. 